\title{
Detection of Motorbike Speed Estimates Using Moving Object Detection with Frame Difference
}

\author{
Eva Y. Puspaningrum ${ }^{1}$, M. Syahrul Munir, Wahyu S.J. Saputra, Haris Ardianto W. \\ Informatics Engineering \\ Universitas Pembangunan Nasional "Veteran" Jawa Timur \\ Surabaya, Indonesia \\ 1evapuspaningrum.if@upnjatim.ac.id
}

\begin{abstract}
The application of technology has developed in the field of traffic. The occurrence of traffic violations and an increase in the number of accidents triggered by the speed of vehicles that exceed the maximum limit specified. It especially two-wheeled vehicle or motorbike. The small form of a motorbike most often violates with speed exceeding the maximum limit.

In this research discuss the detection of motorbike speed. The data used is video. This speed detection uses moving object detection with frame difference. In this method, the video will be cut into a frame image that will be detected. For each frame image obtained will be compared to any moving object. From the moving image it will be used to calculate the distance of the moving object so that the estimated speed of the motorbike.

The results of detection of the distance of the object's displacement and the time used for moving objects can be calculated the average speed of the motorbike. Based on the results of testing the data obtained the average computational value of the program algorithm that for every one frame requires \pm 0.837 seconds. The results of testing the data obtained the MSE value of 8.91 and standard error of $\pm 0.6 \mathrm{~km} / \mathrm{hour}$.
\end{abstract}

Keywords-motorbike speed estimation; moving object detection; frame difference.

\section{INTRODUCTION}

Traffic conditions are increasingly congested resulting in many traffic violations. This is exacerbated by how to drive that is not by the rules, one of which is the speed of the vehicle users that are prone to accidents. It especially two-wheeled vehicle or motorbike. The small form of a motorcycle most often violates with speed exceeding the maximum limit. Then it is necessary to make efforts to curb this dangerous behavior. Therefore an application was made to detect the speed of the motor vehicle. So that the traffic officers who are on guard can find out how fast each motorbike can be acted upon. So speed observation by officers is very important to help control traffic users.

In particular, vehicle detection on the road is a topic that is very interesting for researchers over the past decade [1]. A variety of sensing modalities has become available for on-road vehicle detection. Imaging technology has been greatly developed in recent years. Cameras are cheaper, smaller and higher quality than before. Vehicle detection on the road also requires a faster process than other applications because the speed of the vehicle is limited by the level of processing [2] Another issue is the robustness of vehicle movements. Several special issues have also focused on computer vision applications in intelligent transportation systems such as object detection.

Moving object detection technology distinguishes moving objects and backgrounds, and extracts moving targets from digital video which traffics, roads, residential and other intelligent monitoring systems. The results of moving object detection will directly affect the effect of moving target tracking [3]. Detection of moving objects from a video is an important part, because it can be used in many areas such as intelligent video surveillance, motion analysis, etc. According to research conducted by Mishra et al. [4], there are three methods commonly used for moving object detect. These methods are background subtraction, optical flow and temporal differences. Background subtraction serves to compare certain images with images that are used as references. Optical flow is a motion segmentation method that uses movement characteristics to detect motion passing through a camera. This method requires complex computational processes and is very sensitive to interference [5]. Temporal differences method is also known as frame difference. This method is done by comparing the captured image frames according to the time sequence

Moving object detection is the basis of move object identification and tracking. Even though a lot of research has been done in recent years, the subject is still challenging. The position of the moving object is detected from the difference in frame two frames in a row [6]. Frame-difference methods have been used widely for moving target detection [7]. Motion detection algorithms include the frame difference method, background reduction method, optical flow method and statistical learning method [8]. Optical flow method is the method that consumes the most computational time than other methods because the algorithm is complex. Statistical learning methods also have a lot of computational complexity because they require many training samples. Both of these methods are not suitable for real-time processing. The background reduction method is susceptible to lighting. The frame difference method is simple to implement. This algorithm increases the segmentation and location of objects. It has a good detection speed and a small computing process, so this method is right to use for real-time processing. To detect moving objects in surveillance videos captured by immovable cameras, the simplest method is frame difference method because of the reason it has great detection speed, can be implemented on hardware easily and has been used widely [9]. 
Objects that move in video recordings can be known for their speed by using a particular technique. The moving object can be calculated by using a sequence of video frames. This technique works by comparing images to get information that determines whether or not there is a movement. In this paper will be discussed about the detection of motorcycle speed estimates by utilizing the movement of moving objects using the frame difference method. Frame difference method is a simple form of background reduction. The process of this method only reduces the current frame with the previous frame along the number of video frame sequences. In this study, the average motorbike speed of a video will be obtained in realtime.

\section{Methodology}

The design process to obtain the estimated speed value there are two stages, namely motion detection and speed calculation. The process can be seen in Fig. 1.

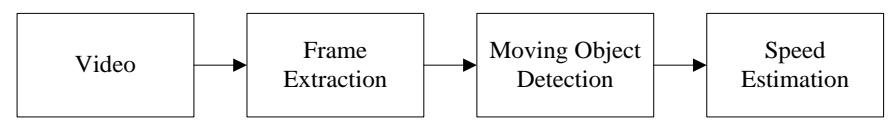

Fig. 1. The Design Process Of Speed Estimation

\section{A. Frame Extraction}

At this stage, the frame difference method can be used to separate several parts of the picture frame from the video obtained. This aims to obtain the movement of images or objects in the image. Where at this stage between background and object will be detected changes in each frame. To be able to cut a video into several picture frames that is by using the video cutting technique based on the number of frames in 1 second (FPS) in the video that has been recorded. In another word it can be illustrated as follows, in 1 second the video data (t) that is recorded can produce $30 \mathrm{fps}$. So if within 2 seconds the video record can provide 60 frames of images. The number of images obtained in one video can be seen from formula (1).

$$
A=t * 30 f p s
$$

$A$ is the number of images and $t$ is the time per second.

\section{B. Moving Object Detection}

One method in moving object detection is to use the movement of objects in each frame. One of them is a method called frame difference. Frame Difference method performs through 3 stages shown in Fig. 2. The first stage is the process of determining the background image. The second stage is the subtraction process, and the last is the threshold. The threshold is an important part for assessing the accuracy of motion detection [10].

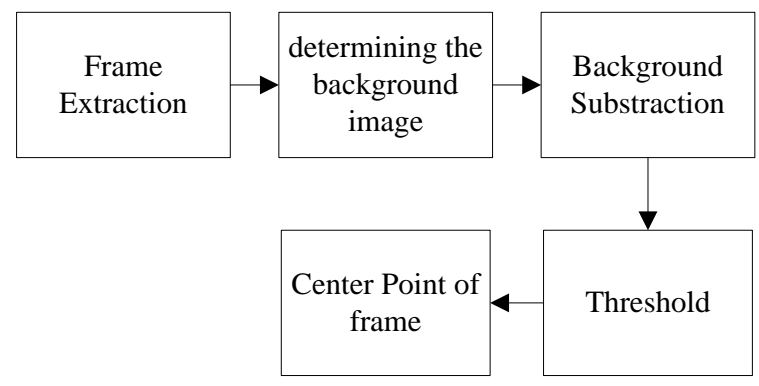

Fig. 2. Process of Frame Difference

To detect moving objects in a video taken with a camera that cannot be moved, this is so that the background used is not replaced so that only the moving object is obtained. In determining the background can be done using the mode value of each frame that is processed. Then the output obtained is the pixel value of the background matrix of all frames that are processed. The specified background will be compared between the frame and the background to obtain a frame difference. The main goal is to know the moving object by interpreting the value of each pixel from the frame image.

Background Subtraction aims to detect/take the foreground from the background for further processing. Background search results by searching for the mode values of each frame. The collected mode values will be grouped and formed into RGB images so that they create a complete image named Background image.

The threshold is used to adjust the amount of gray in the image. With this stage, the results of the gray degree that exceeds the limit will be rounded to a value of 1 and a smaller gray degree will be given a value of 0 . This is done to make it easier to determine the distance between frames that has obtained the center point of the frame image. After the threshold and obtain a binary image, the next step is to find the center point of each frame. This aims to make it easier to find the distance of movement of objects between each frame. The process of determining the center point of the frame can be seen in Fig. 3.

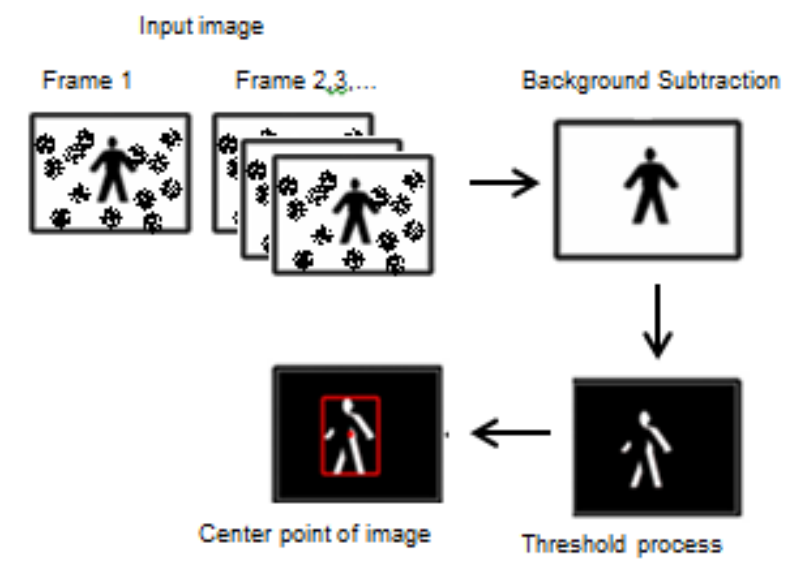

Fig. 3. Determine the Center Point of Frame 


\section{Speed Estimation}

To get the results of the estimation of the speed of the motorbike can use the mathematical equation to calculate the speed of the vehicle based on the displacement of the object and the time of the transfer trip. In this case, the input data used is the result of the separation of video images used as frames. Furthermore, the data frame will be detected by changing the object changes by taking the $n$ value of all frames shown in Fig. 4.

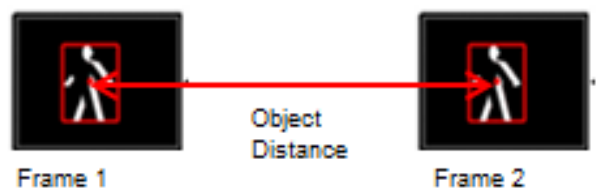

Fig. 4. Distance of the Object's Center Point

After obtaining the inter-frame distance as in Fig. 4, it can be calculated the estimated speed of the detected vehicle. Using the equation to calculate speed is shown in (2).

$$
v=\frac{s}{t}
$$

$v$ is calculating speed, $s$ is distance, $t$ is the time of object displacement. The object values of the objects stored in an array variable are calculated using the Euclidean; this equation formula can be calculated based on the coordinate points of the resulting center point [11]. Furthermore, if the Euclidean distance value has been obtained in the form of the moving object distance, the estimated speed of the motor vehicle will be calculated. From the equation to be able to calculate there is a distance of 1 pixel which means that every one px represents how much the actual distance meter scale is in meters. The calculation is shown in (3).

$$
v=\frac{\text { Euclidean distance } * \text { distance } 1 \text { px } * \text { fps } * 3600}{\text { total of frame } * 1000}
$$

\section{EXPERIMENTAL EVALUATION}

The data that will be used in this study is data from video recordings of motorcycle movements. The specifications of the data used are shown in Table I.

TABLE I. DATA SPECIFICATION

\begin{tabular}{|l|c|}
\hline Format & $* . m p 4, * . a v i$ \\
\hline Resolution & SD / 360 x 640 \\
\hline fps & 30 \\
\hline Video capture angle & $\begin{array}{c}\text { Parallel objects }>30^{\circ} \\
\text { to }<90^{\circ}\end{array}$ \\
\hline Duration & $<=6$ second \\
\hline
\end{tabular}

Next step after determining the data specification is the separation of each frame from the video image used. This stage is the initial stage to be able to do the frame difference method. When cutting frames from video data, the first thing to do is to determine the number of FPS from the data and the duration of the data so that it can get the total number of frames.

Fig. 5 shows several frames from a video. In Fig. 5 can be seen in frames number 27, 28, and 29 and frames 40, 41 and 42. This means that on frame $30-39$ there is a movement of a motorbike.

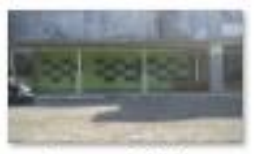

Frame 27.jpg

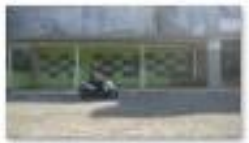

Frame 40.jpg

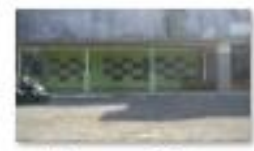

Frame 28.jpg

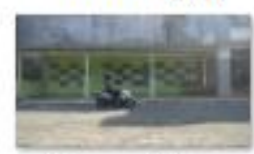

Frame 41.jpg

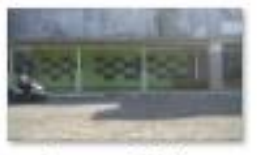

Frame 29.jpg

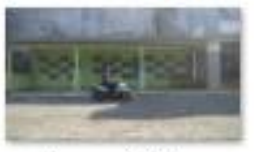

Frame 42.jpg
Fig. 5. Some of Frames from a Video

After separating each part of the frame, the next step is to do a background search of the entire frame. Video background is searched using the mode value of each pixel on the frame. Mode values collected will be grouped and formed into an image to form a complete image named background image. The background image can be seen in Fig. 6 .

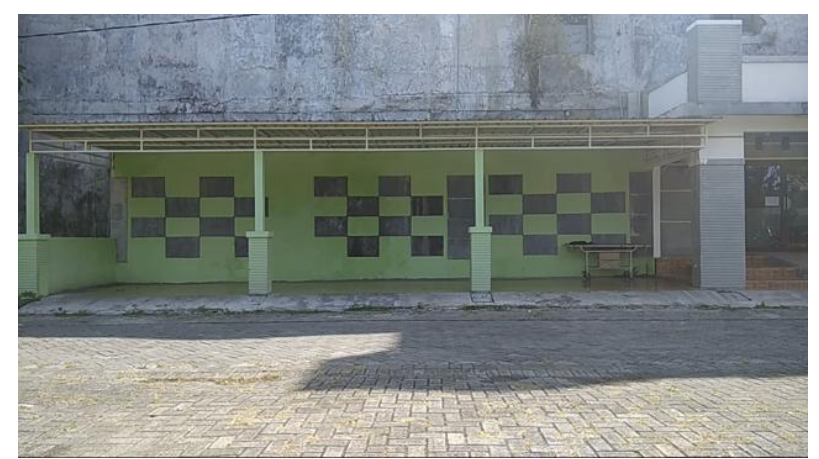

Fig. 6. Background Image

The next stage after obtaining a background image, the frame difference process can be done. At this stage it also processes the image threshold, creates bounding boxes, and looks for the midpoint of the object. To be able to do the frame difference process by reducing the currentFrame value or the stored frame image will be cut by the background image.

The subtraction results from the currentFrame with the background can be seen in the Fig. 7. If there is a reduction where the reduced value is not close to 0 then the result of the subtraction gets the result of a fairly high gray value. So that the pixel that has a high gray value will be concluded that it is an object. 


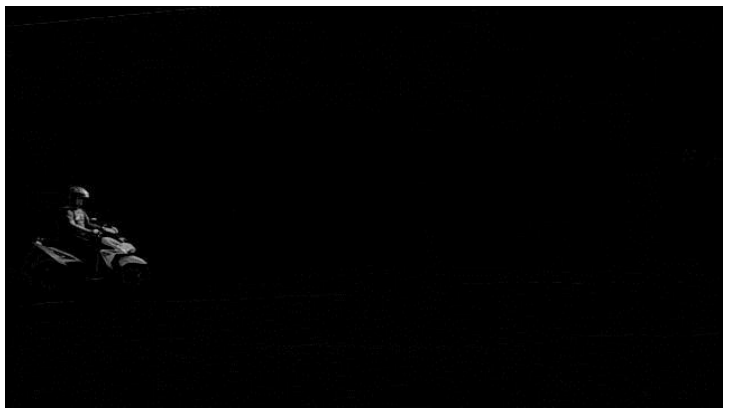

Fig. 7. Subtraction Result

Each object in each frame is used as a moving object if the object results in pixel displacement for each object that has a high gray value.

To provide a better level of object accuracy, a thresholding process is carried out. This is so that the resulting object looks more real and detailed objects. This result can be seen in Fig. 8 .

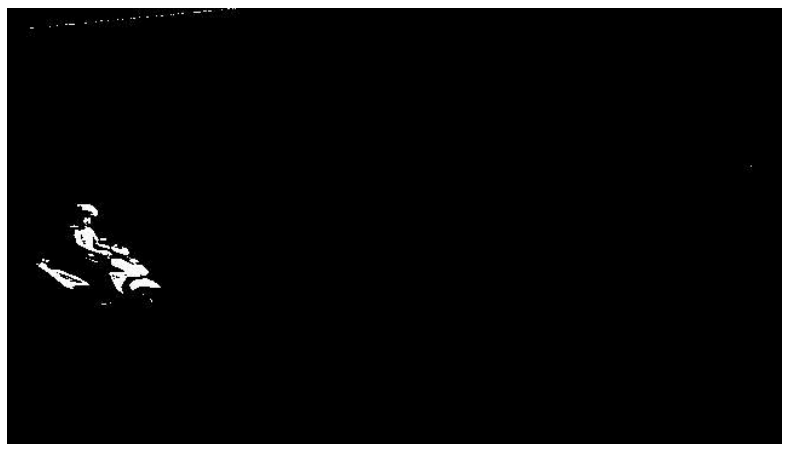

Fig. 8. Thresholding Result

If the thresholding process feels that the object obtained is not good then it can be done with a morphological operation. This process will provide two possibilities that occur, namely thickening object details or reducing object details. The intensity of the changing light or white balance is very influential on the determination of objects, where the value obtained if there is a change in white balance is noise. So that the noise-noise will affect the object detection, in other words the captured noise will be made as an object at once.

After the threshold process, the next thing to be determined is the coordinates of the object. The coordinates will be saved and displayed in a bounding box plot. The next stage is that the object detected by the bounding box will find the center point of the object. The center point value is what will be used as a reference that the object moves each pixel.

The center point of the object that is obtained will be calculated as the moving distance so that the value of the object's displacement distance can be known.

In Fig. 9 shows the moving object of a motorcycle that is given a red bounding box.

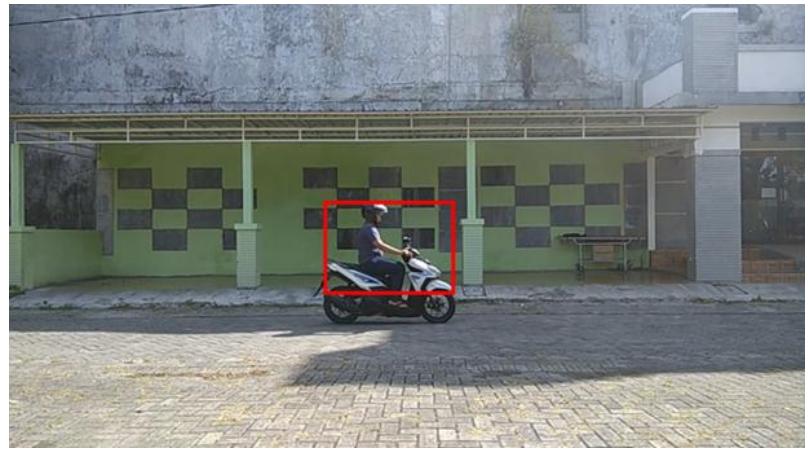

Fig. 9. Bounding Box Object

From several experiments using an average speedometer and GPS speed of $<50 \mathrm{~km} / \mathrm{h}$, the distance of the path traveled by a vehicle of 13 meters produces data in Table II.

TABLE II. TESTING RESULT

\begin{tabular}{|c|c|c|c|c|c|}
\hline No. & $\begin{array}{c}\text { Program } \\
\text { speed of } \\
\text { km / hour }\end{array}$ & $\begin{array}{c}\text { Speedom } \\
\text { eter } \\
\text { speed } \\
\text { km/hour }\end{array}$ & $\begin{array}{c}\text { Speedom } \\
\text { eter error } \\
(\mathbf{x})\end{array}$ & $\begin{array}{c}\text { GPS } \\
\text { speed } \\
\text { Km/hour }\end{array}$ & $\begin{array}{c}\text { GPS } \\
\text { error } \\
\text { difference } \\
(\mathbf{x})\end{array}$ \\
\hline 1 & 19.7975 & 20 & 0.2025 & 18 & 1.7975 \\
\hline 2 & 30.2735 & 30 & 0.2735 & 27 & 3.2735 \\
\hline 3 & 31.4502 & 40 & 8.5498 & 37 & 5.5498 \\
\hline 4 & 45.9232 & 50 & 5.1704 & 47 & 2.1703 \\
\hline 5 & 23.3836 & 30 & 6.6164 & 27 & 3.6164 \\
\hline 6 & 27.9243 & 35 & 7.0757 & 33 & 5.0757 \\
\hline 7 & 16.4983 & 20 & 3.5017 & 18 & 1.5017 \\
\hline 8 & 25.3235 & 30 & 4.6765 & 27 & 1.6765 \\
\hline 9 & 33.9158 & 40 & 6.0842 & 37 & 3.0842 \\
\hline 10 & 18.1078 & 20 & 1.8922 & 18 & 0.1078 \\
\hline 11 & 26.591 & 30 & 3.409 & 27 & 0.409 \\
\hline 12 & 32.9253 & 40 & 7.0747 & 37 & 4.0747 \\
\hline 13 & 15.7478 & 20 & 4.2522 & 18 & 2.2522 \\
\hline 14 & 15.9766 & 20 & 4.0234 & 18 & 2.0234 \\
\hline 15 & 16.328 & 20 & 3.672 & 18 & 1.672 \\
\hline 16 & 25.4 & 30 & 4.6 & 27 & 1.6 \\
\hline 17 & 32.0744 & 40 & 7.9256 & 37 & 4.9256 \\
\hline 18 & 44.9293 & 50 & 5.0707 & 47 & 2.0707 \\
\hline
\end{tabular}

Table II shows for each speed test obtaining varying values. The error difference value is based on the benchmark on the Speedometer and GPS. For the error difference value obtained, it will be analyzed based on the statistical approach using MSE, Standard Deviation, and Standard Error.

In statistics is a value expected from the squared error. Existing errors indicate how big the estimation results are different from the values to be estimated. The difference occurs because of randomness to the data or because the estimator does not contain information that can produce a more accurate 
estimate, here is an equation to calculate the MSE value. MSE formula can be seen in formula (4).

$$
\operatorname{MSE}=\frac{1}{N} \sum_{t=h}^{N}\left(y_{t}-\tilde{y}_{t}\right)^{2}
$$

$\mathrm{N}$ is the amount of data, $y_{t}$ is the actual value of the index or benchmark value and $\tilde{y}_{t}$ is a predictive value or test result value.

For calculation results, the error value based on the speedometer is obtained MSE $=25.617$, Standard Deviation = 2.612 and standard error $=0.6$. For the calculation value of the error difference value based on GPS obtained MSE 8.910, Standard Deviation $=2.432$ and standard error 0.57 .

To find out the performance of the program, the computational value for each processed data will be calculated. Then the computational value is obtained in Table III.

TABLE III. COMPUTING TIME

\begin{tabular}{|c|c|c|c|}
\hline No. & $\begin{array}{c}\text { Computing } \\
\text { time } \\
\text { (seconds) }\end{array}$ & $\begin{array}{c}\text { Number } \\
\text { of } \\
\text { Frames }\end{array}$ & $\begin{array}{c}\text { One frame } \\
\text { computing } \\
\text { time } \\
\text { (seconds) }\end{array}$ \\
\hline 1 & 110 & 131 & 0.839 \\
\hline 2 & 80 & 96 & 0.833 \\
\hline 3 & 105 & 120 & 0.875 \\
\hline 4 & 172 & 151 & 1.139 \\
\hline 5 & 143 & 164 & 0.871 \\
\hline 6 & 79 & 107 & 0.738 \\
\hline 7 & 139 & 168 & 0.827 \\
\hline 8 & 140 & 163 & 0.858 \\
\hline 9 & 76 & 106 & 0.716 \\
\hline 10 & 159 & 176 & 0.903 \\
\hline 11 & 119 & 139 & 0.856 \\
\hline 12 & 120 & 143 & 0.839 \\
\hline 13 & 137 & 156 & 0.878 \\
\hline 14 & 111 & 132 & 0.84 \\
\hline 15 & 118 & 142 & 0.83 \\
\hline 16 & 76 & 109 & 0.697 \\
\hline 17 & 66 & 98 & 0.673 \\
\hline 18 & 122 & 142 & 0.859 \\
\hline
\end{tabular}

Table III shows the computation time for each video data that is executed in the speed estimation program. For each data executed in the compiled algorithm, the average computational value is 0.837 seconds. So it can be concluded that the average computational value of the program algorithm that for every one frame requires \pm 0.837 seconds.

\section{CONCLUSION}

Frame difference is a method for detecting the motion of objects that is easy and simple but has several weaknesses and advantages. To detect moving objects there are several things that must be considered, namely the level of light intensity because with changes in light intensity, especially white balance, the detection of objects cannot run properly due to the emergence of some noise image that appears. The color of the object to be detected is the main effect of determining the object because when the object difference process is carried out the object changes can change the size and coordinates of the center point of the object. Based on the results of the tests that have been carried out, it is obtained the MSE value of 8.910 and the standard error of $\pm 0.6 \mathrm{~km} /$ hour. Factors that affect the system are the presence of several movements in the background other than the main object. The computing time for every 1 frame in the data requires \pm 0.837 seconds.

\section{ACKNOWLEDGMENT}

The authors thank the University of Pembangunan Nasional "Veteran" Jawa Timur, especially for Informatics Engineering Department and Faculty of Computer Science for providing financial support for this Research.

\section{REFERENCES}

[1] S. Chien, S. Ma, and L. Chen, "Efficient moving object segmentation algorithm using background registration technique," Circuits and Systems for Video, vol. 12, no. 7, pp. 577-586. 2002.

[2] S. Mishra, P. Mishra, N.K. Chaudhary, and P. Asthana, "A Novel Comprehensive Method for Real Time Video Motion Detection Surveillance,” Int. J. Sci. Eng. Res., vol. 2, April 2011

[3] S. Murali and R. Girisha, "Segmentation of motion objects from surveillance video sequences using temporal differencing combined with multiple correlation," 6th IEEE Int. Conf. Adv. Video Signal Based Surveill., pp. 472-477, AVSS 2009.

[4] P. Rosin and T. Ellis, "Image Difference Threshold Strategies and Shadow Detection". Proc. Br. Mach. Vis. Conf., pp. 35_1-35_10, 1995.

[5] S. Sivaraman and M.M. Trivedi, "Looking at vehicles on the road: A survey of vision-based vehicle detection, tracking, and behavior analysis". IEEE Trans. Intell. Transport. Syst., vol. 14, pp. 1773-1795, 2013.

[6] Z. Sun, G. Bebis, and R. Miller, "On-road vehicle detection: A review". IEEE Trans. Pattern Anal. Mach. Intell., vol. 28, pp. 694-711, 2006.

[7] H. Yin, Y. Chai, S.X. Yang, and X. Yang, "Fast-moving target tracking based on mean shift and frame-difference methods," J. Syst. Eng. Electron., vol. 22, pp. 587-592. 2011.

[8] C. Zhan, X. Duan, S. Xu, Z. Song, and M. Luo, "An Improved Moving Object Detection Algorithm Based on Frame Difference and Edge Detection," Fourth Int. Conf. Image Graph., pp. 519-523, 2007.

[9] M. Ren and H. Sun. "A practical method for moving target detection under complex background," J. Comp. Eng., vol. 20, pp. 33-34, 2005.

[10] Y. Zhang, X. Wang, and B. Qu, "Three-frame difference algorithm research based on mathematical morphology," Proc. Eng., vol. 29, pp. 2705-2709, 2011.

[11] K. Sirisha, and R Patnaik, "Implementation of Moving Object Tracking and Determination of Velocity Using DSK". Int. J. Sci. Eng. Res., vol. 3, pp. 2229-5518, November 2012 . 